\title{
Teaching NeuroImages: The Motor Band Sign in Amyotrophic Lateral Sclerosis
}

Joshua Budhu, MD, Joseph Rosenthal, MD, PhD, Erika Williams, MD, PhD, and Tracey Milligan, MD

Neurolog ${ }^{\circledR}$ 2021;96:e1092-e1093. doi:10.1212/WNL.0000000000010848

Figure Brain MRI Without Contrast

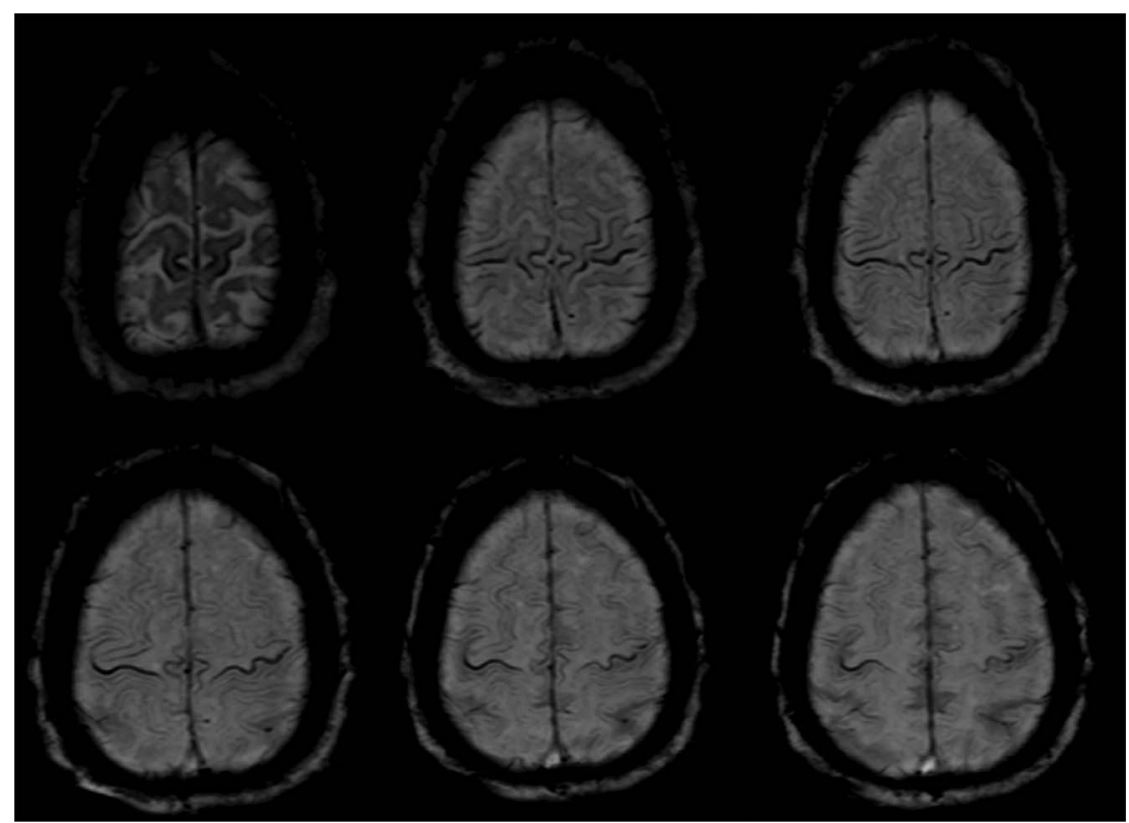

Susceptibility-weighted imaging (SWI) on 1.5 Tesla MRI demonstrating bilateral symmetric hemosiderin deposition along the central sulcus, layering the precentral gyrus.

A 71-year-old woman presented with several months of progressive lower extremity weakness, followed by bulbar weakness. On examination, she had diffuse muscle atrophy, fasciculations, weakness, and hyperreflexia. A diagnosis of clinically definite ALS was made using the El Escorial criteria. At the time of presentation and imaging, she had both severe upper and lower motor neuron involvement. Her ALSFRS-R score was 15, indicating advanced disease. Her brain MRI showed increased signal on susceptibility-weighted imaging, consistent with superficial siderosis along the central sulcus (figure). This finding has been named the "motor band sign". The intracellular iron may be from microglial phagocytosis of degenerated neurons in the motor strip. ${ }^{2}$ Careful scrutiny of susceptibility-weighted imaging should be performed when considering motor neuron disease, as this can be helpful in making the diagnosis.

\section{Study Funding}

No targeted funding reported.

\section{Correspondence}

Dr. Budhu

jbudhu@mgh.harvard.edu

\section{MORE ONLINE}

$\rightarrow$ Teaching slides

links.lww.com/WNL/

B221 


\section{Disclosures}

The authors report no disclosures. Go to Neurology.org/N for full disclosures.

\section{Appendix Authors}

\begin{tabular}{lll}
\hline Name & Location & Contribution \\
\hline $\begin{array}{l}\text { Joshua } \\
\text { Budhu, MD }\end{array}$ & $\begin{array}{l}\text { Brigham and Women's Hospital, } \\
\text { Harvard Medical School, } \\
\text { Boston, MA }\end{array}$ & $\begin{array}{l}\text { Conception, writing, } \\
\text { critical revision, and } \\
\text { final approval of the } \\
\text { text and images }\end{array}$ \\
\hline $\begin{array}{l}\text { Joseph } \\
\text { Rosenthal, } \\
\text { MD, PhD }\end{array}$ & $\begin{array}{ll}\text { Brigham and Women's Hospital, } \\
\text { Harvard Medical School, }\end{array}$ & $\begin{array}{l}\text { Critical revision and } \\
\text { final approval of all } \\
\text { text and images }\end{array}$
\end{tabular}

Appendix (continued)

\begin{tabular}{lll}
\hline Name & Location & Contribution \\
\hline $\begin{array}{l}\text { Erika } \\
\text { Williams, } \\
\text { MD, PhD }\end{array}$ & $\begin{array}{l}\text { Brigham and Women's Hospital, } \\
\text { Harvard Medical School }\end{array}$ & $\begin{array}{l}\text { Critical revision and } \\
\text { final approval of all } \\
\text { text and images }\end{array}$ \\
\hline $\begin{array}{l}\text { Tracey } \\
\text { Milligan, MD }\end{array}$ & $\begin{array}{l}\text { Brigham and Women's Hospital, } \\
\text { Harvard Medical School, } \\
\text { Boston, MA }\end{array}$ & $\begin{array}{l}\text { Critical revision and } \\
\text { final approval of all } \\
\text { text and images }\end{array}$ \\
\hline
\end{tabular}

\section{References}

1. Chakraborty S, Gupta A, Nguyen T, Bourque P. The "motor band sign:" susceptibilityweighted imaging in amyotrophic lateral Sclerosis. Can J Neurol Sci 2015;42:260-263.

2. Kwan JY, Jeong SY, van Gelderen P, Deng H, Quezado MM, Danielian LE, et al. Iron accumulation in deep cortical layers accounts for MRI signal abnormalities in ALS: correlating 7 tesla MRI and pathology. PLoS One 2012;7. doi: 10.1371/journal.pone.0035241. 


\section{Neurology}

Teaching NeuroImages: The Motor Band Sign in Amyotrophic Lateral Sclerosis
Joshua Budhu, Joseph Rosenthal, Erika Williams, et al.

Neurology 2021;96;e1092-e1093 Published Online before print September 14, 2020

DOI 10.1212/WNL.0000000000010848

This information is current as of September 14, 2020

\section{Updated Information \&} Services

References

Subspecialty Collections

Permissions \& Licensing

Reprints including high resolution figures, can be found at: http://n.neurology.org/content/96/7/e1092.full

This article cites 2 articles, 0 of which you can access for free at: http://n.neurology.org/content/96/7/e1092.full\#ref-list-1

This article, along with others on similar topics, appears in the following collection(s):

All Clinical Neurology

http://n.neurology.org/cgi/collection/all_clinical_neurology Amyotrophic lateral sclerosis

http://n.neurology.org/cgi/collection/amyotrophic_lateral_sclerosis_ Anterior nerve cell disease

http://n.neurology.org/cgi/collection/anterior_nerve_cell_disease MRI

http://n.neurology.org/cgi/collection/mri

Information about reproducing this article in parts (figures,tables) or in its entirety can be found online at:

http://www.neurology.org/about/about_the_journal\#permissions

Information about ordering reprints can be found online:

http://n.neurology.org/subscribers/advertise

Neurology ${ }^{\circledR}$ is the official journal of the American Academy of Neurology. Published continuously since 1951, it is now a weekly with 48 issues per year. Copyright () 2020 American Academy of Neurology. All rights reserved. Print ISSN: 0028-3878. Online ISSN: 1526-632X.

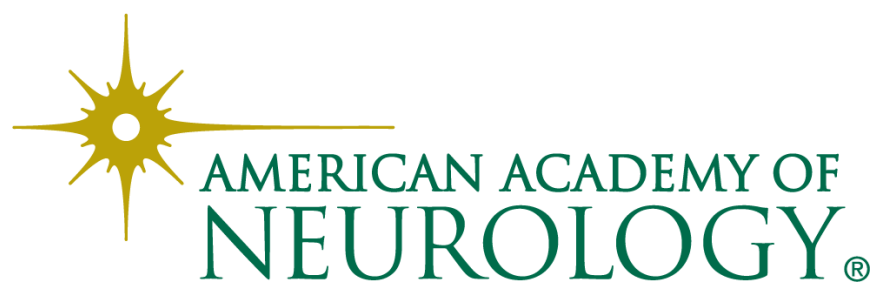

